\title{
Perfil epidemiológico de acidentes ofídicos do Estado do Amapá
}

\section{Epidemiological profile of snake poisoning accidents in the State of Amapá}

\author{
Ana Cristina Silva Ferreira Lima ${ }^{1}$, Carlos Eduardo Costa Campos ${ }^{1}$ \\ e José Renato Ribeiro²
}

\begin{abstract}
RESUMO
0 presente trabalho apresenta o perfil epidemiológico dos acidentes ofídicos notificados junto à Secretaria do Estado de Saúde do Estado do Amapá. Para isso, foram analisadas 909 fichas durante o período de 2003 a 2006. A maior frequiência de picadas foi no ano de 2004 com registro de 255 casos, seguido pelo ano de 2006 com 246 casos. Os números mais expressivos de pacientes encontram-se nas faixas etárias compreendidas entre 20 e 34 anos (30\%). Os gêneros Bothrops e Crotalus foram responsáveis por 67,5\% e 0,7\% dos acidentes, respectivamente. Serpentes consideradas não peçonhentas causaram $0,2 \%$ dos acidentes e em $31,2 \%$ dos casos não foi possível a identificação da espécie. Indivíduos do sexo masculino (80,6\%) foram os mais atingidos. A maior incidência de picadas foi nos membros inferiores (68\%). Os acidentes ocorreram, em sua maioria, na área rural (62,7\%), em circunstâncias equilibradas de trabalho (60\%) e lazer (15,6\%). 0 tempo decorrido entre a picada e 0 atendimento ao paciente foi de 12 horas (29\%). Dos acidentes ofídicos, 263 foram classificados como leves, 193 graves e 187 moderados.
\end{abstract}

Palavras-chaves: Epidemiologia. Serpentes peçonhentas. Ofidismo.

\section{ABSTRACT}

This study presents the epidemiological profile of snake poisoning accidents notified to the Health Department of the State of Amapá. For this, 909 records over the period from 2003 to 2006 were analyzed. The greatest frequency of bites was in the year 2004, with 255 cases recorded, followed by the year 2006 with 246 cases. The largest numbers of patients were in the age range between 20 and 34 years (30\%). The genera Bothrops and Crotalus accounted for $67.5 \%$ and $0.7 \%$ of the accidents, respectively. Snakes that are considered non-venomous caused $0.2 \%$ of the accidents, and it was not possible to identify the species in $31.2 \%$ of the cases. Male individuals were more affected (80.6\%). The highest incidence of bites was on the lower limbs (68\%). The accidents mostly occurred in rural areas $(62.7 \%)$, during balanced work circumstances $(60 \%)$ or leisure activities $(15.6 \%)$. The time elapsed from the bite to attending the patient was 12 hours (29\%). Among the snake poisoning accidents, 263 were classified as mild, 187 as moderate and 193 as serious.

Key-words: Epidemiology. Venomous snakes. Ophidism.

Os acidentes ofídicos representam sério problema de saúde pública nos países tropicais, pela freqüência com que ocorrem e pela morbi-mortalidade que ocasionam ${ }^{19}$. Na América do Sul, o Brasil é o país com maior número de acidentes com cerca de 20.000 casos por ano, seguido pelo Peru (4.500), Venezuela (2.500 a 3.000), Colômbia (2.675), Equador (1.200 a 1.400) e Argentina (1.150 a 1.250) ${ }^{26}$. No Brasil, as regiões com maior incidência de acidentes ofídicos por 100.000 habitantes são as regiões centro-oeste e norte ${ }^{4}$. No entanto, é possível que haja um elevado índice de subnotificações na região Norte, uma vez que há grande dificuldade de acesso aos serviços de saúde ${ }^{4}$.

A maior parte dos acidentes causados por serpentes peçonhentas, no Brasil, é atribuída ao gênero Bothrops, que

1. Laboratório de Zoologia, Departamento de Ciências Biológicas, Universidade Federal do Amapá, Macapá, AP. 2. Secretaria de Saúde do Estado do Amapá, Ministério da Saúde, Macapá, AP.

Endereço para correspondência: Dr. Carlos Eduardo Costa Campos. Dept ${ }^{0}$ de Ciências Biológicas/UNIFAP. Rodovia Juscelino Kubitschek, Km 02, Bairro Jardim Marco Zero, 68902-280 Macapá, AP.

e-mail: ceccampos@unifap.br

Tel: $55963312-1700$

Recebido para publicação em 16/12/2008

Aceito em 29/04/2009 representam 90\% das espécies envolvidas neste tipo de acidente, seguidos pelos gêneros Crotalus 7,7\%, Lachesis 1,4\% e Micrurus $0,5 \%{ }^{14}$. Em relação às serpentes não peçonhentas, existem algumas espécies que são consideradas de importância médica e que poderiam causar um suposto envenenamento sistêmico ou fatal, como é o caso dos gêneros Phalotris, Philodryas, Xenodon e Tachimenis ${ }^{2426}$.

Os acidentes ofídicos têm importância médica em virtude da sua frequiência e gravidade. Desta forma, estudos sobre os acidentes ofídicos com a notificação da correta identificação dos animais causadores destes acidentes são de extrema relevância, pois subsidiam melhores condições de atendimento e tratamento dos $\operatorname{acidentados}^{912}$.

\section{MATERIAL E MÉTODOS}

Neste trabalho, foram coletadas informações junto à Secretaria Estadual de Saúde do Estado do Amapá sobre acidentes ofídicos registrados durante o período de 2003 a 2006, constantes no Programa do Sistema de Informação de Agravos e de Notificações (SINAN) do Ministério da Saúde. 
Das notificações, foram obtidos dados referentes ao sexo dos pacientes, gênero de serpente, época do ano, óbito, a idade da vítima, local da picada, tempo decorrido entre o acidente e o atendimento, ocupação do paciente e a localidade dos acidentes.

\section{RESULTADOS}

Foram notificados à Secretaria Estadual de Saúde, nos anos de 2003, 2004, 2005 e 2006, respectivamente, 193, 255, 215 e 246 acidentes por serpentes peçonhentas ocorridos no Estado do Amapá, totalizando 909 acidentes, com média de 227,3 $\pm 27,7$ (Figura 1).

Quanto à sazonalidade, os meses de janeiro e abril foram os de maior incidência de acidentes ofídicos, apresentando $102(11,2 \%)$ e 100 (11\%) dos casos (Tabela 1).

Dos casos avaliados, 80,6\% ocorreram em indivíduos do sexo masculino e 19,3\% em indivíduos do sexo feminino (Tabela 2).
Um total de $570(62,7 \%)$ acidentes ocorreu na zona rural do Estado do Amapá, 271 (30,9\%) na zona urbana e 58 (6,4\%) teve como ignorado o local de ocorrência (Tabela 3).

Os números mais expressivos de acidentes foram observados nas faixas etárias compreendidas entre 20 e 34 anos, com 271 (29,8\%) casos (Figura 2).

As circunstâncias dos acidentes mostraram que $60,1 \%$ dos agravos ocorreram durante atividade relacionada ao trabalho e 15,6\% durante atividades de lazer (Figura 3). As circunstâncias dos acidentes entre as atividades de lazer e trabalho não mostraram diferenças significativas $(\mathrm{p}>0,05)$.

0 reflexo ocupacional que se observa está ligado à faixa etária economicamente ativa (e.g. agricultores e autônomos), perfazendo $68 \%$ dos pacientes picados. 0s estudantes apresentaram um percentual de 20\% (Figura 4).

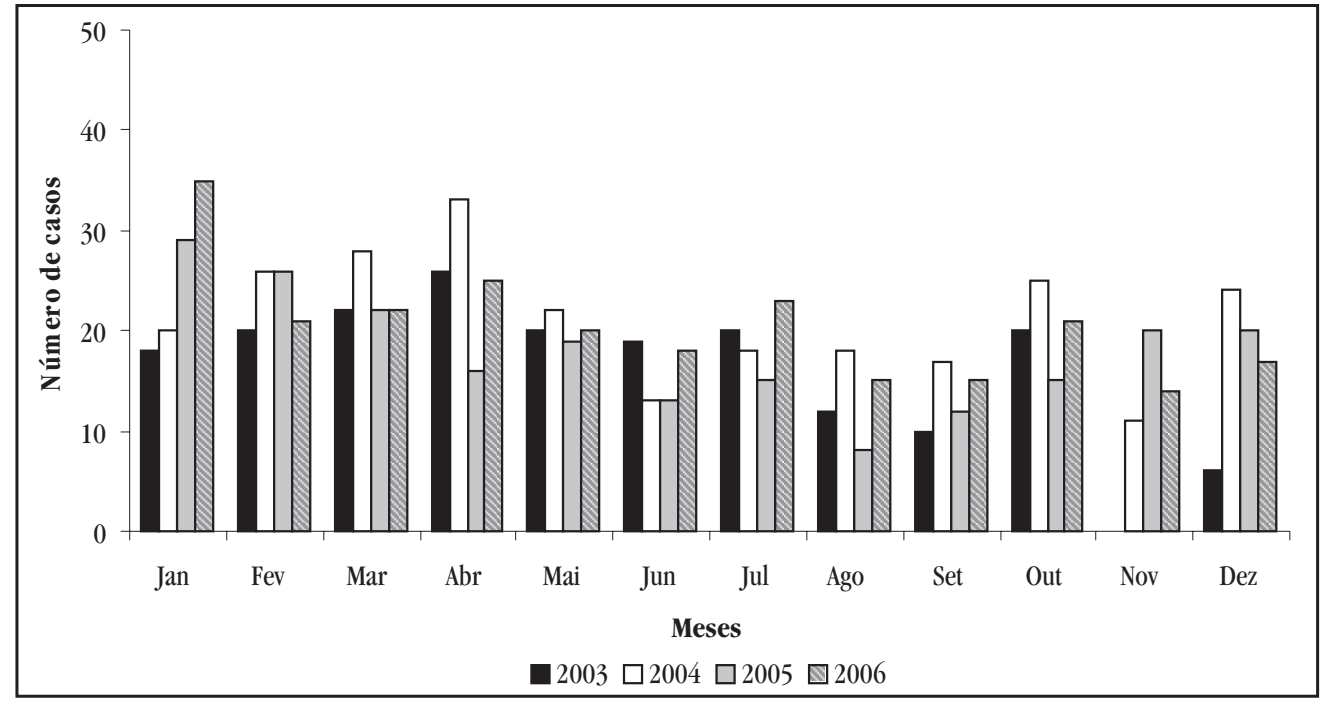

FIGURA 1

Frequência mensal e anual dos acidentes ofídicos no Estado do Amapá, no período de 2003 a 2006.

TABELA 1

Distribuição dos acidentes ofídicos no Estado do Amapá, segundo o mês de ocorrência, no período de 2003 a 2006.

\begin{tabular}{lrrrrrrr}
\hline & \multicolumn{3}{c}{ Ano } & & \multicolumn{2}{c}{ Total } \\
\cline { 2 - 4 } \cline { 7 - 8 } Mês de ocorrência & 2003 & 2004 & 2005 & 2006 & & $\mathrm{n}^{0}$ & $\%$ \\
\hline Janeiro & 18 & 20 & 29 & 35 & & 102 & 11,2 \\
Fevereiro & 20 & 26 & 26 & 21 & & 93 & 10,2 \\
Março & 22 & 28 & 22 & 22 & & 94 & 10,4 \\
Abril & 26 & 33 & 16 & 25 & & 100 & 11,0 \\
Maio & 20 & 22 & 19 & 20 & & 81 & 8,9 \\
Junho & 19 & 13 & 13 & 18 & & 63 & 6,9 \\
Julho & 20 & 18 & 15 & 23 & & 76 & 8,4 \\
Agosto & 12 & 18 & 8 & 15 & & 53 & 5,8 \\
Setembro & 10 & 17 & 12 & 15 & & 54 & 5,9 \\
Outubro & 20 & 25 & 15 & 21 & & 81 & 8,9 \\
Novembro & - & 11 & 20 & 14 & & 45 & 5,0 \\
Dezembro & 6 & 24 & 20 & 17 & & 67 & 7,4 \\
\hline Total & $\mathbf{1 9 3}$ & $\mathbf{2 5 5}$ & $\mathbf{2 1 5}$ & $\mathbf{2 4 6}$ & $\mathbf{9 0 9}$ & $\mathbf{1 0 0 , 0}$ \\
\hline
\end{tabular}

TABELA 2

Distribuição dos acidentes ofídicos no Estado do Amapá, segundo o sexo, no período de 2003 a 2006.

\begin{tabular}{|c|c|c|c|c|c|c|}
\hline \multirow[b]{2}{*}{ Sexo } & \multicolumn{4}{|c|}{ Ano } & \multicolumn{2}{|c|}{ Total } \\
\hline & 2003 & 2004 & 2005 & 2006 & $\mathrm{n}^{0}$ & $\%$ \\
\hline Masculino & 164 & 200 & 181 & 188 & 733 & 80,6 \\
\hline Feminino & 29 & 54 & 34 & 58 & 175 & 19,3 \\
\hline Ignorado & 0 & 1 & 0 & 0 & 1 & 0,1 \\
\hline Total & 193 & 255 & 215 & 246 & 909 & 100,0 \\
\hline
\end{tabular}

TABELA 3

Distribuição dos acidentes ofídicos no Estado do Amapá, segundo a zona de ocorrência, no período de 2003 a 2006.

\begin{tabular}{lrrrrrrr}
\hline & \multicolumn{4}{c}{ Ano } & & \multicolumn{2}{c}{ Total } \\
\cline { 2 - 5 } Zona & 2003 & 2004 & 2005 & 2006 & & $\mathrm{n}^{0}$ & $\%$ \\
\hline Rural & 122 & 138 & 159 & 151 & & 570 & 62,7 \\
Urbana & 47 & 88 & 57 & 89 & & 281 & 30,9 \\
Ignorado & 22 & 29 & 2 & 5 & & 58 & 6,4 \\
\hline Total & $\mathbf{1 9 1}$ & $\mathbf{2 5 5}$ & $\mathbf{2 1 8}$ & $\mathbf{2 4 5}$ & & $\mathbf{9 0 9}$ & $\mathbf{1 0 0 , 0}$ \\
\hline
\end{tabular}




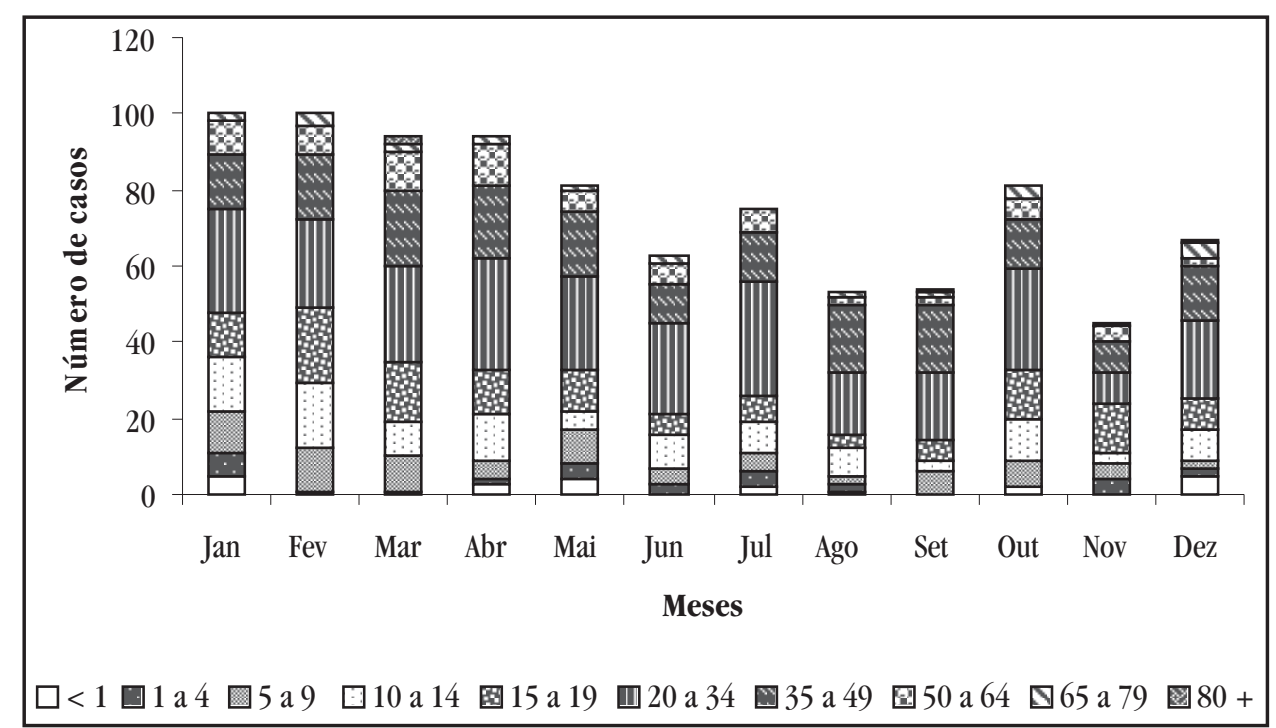

FIGURA 2

Frequência mensal dos acidentes no Estado do Amapá, segundo a faixa etária, no período de 2003 a 2006.

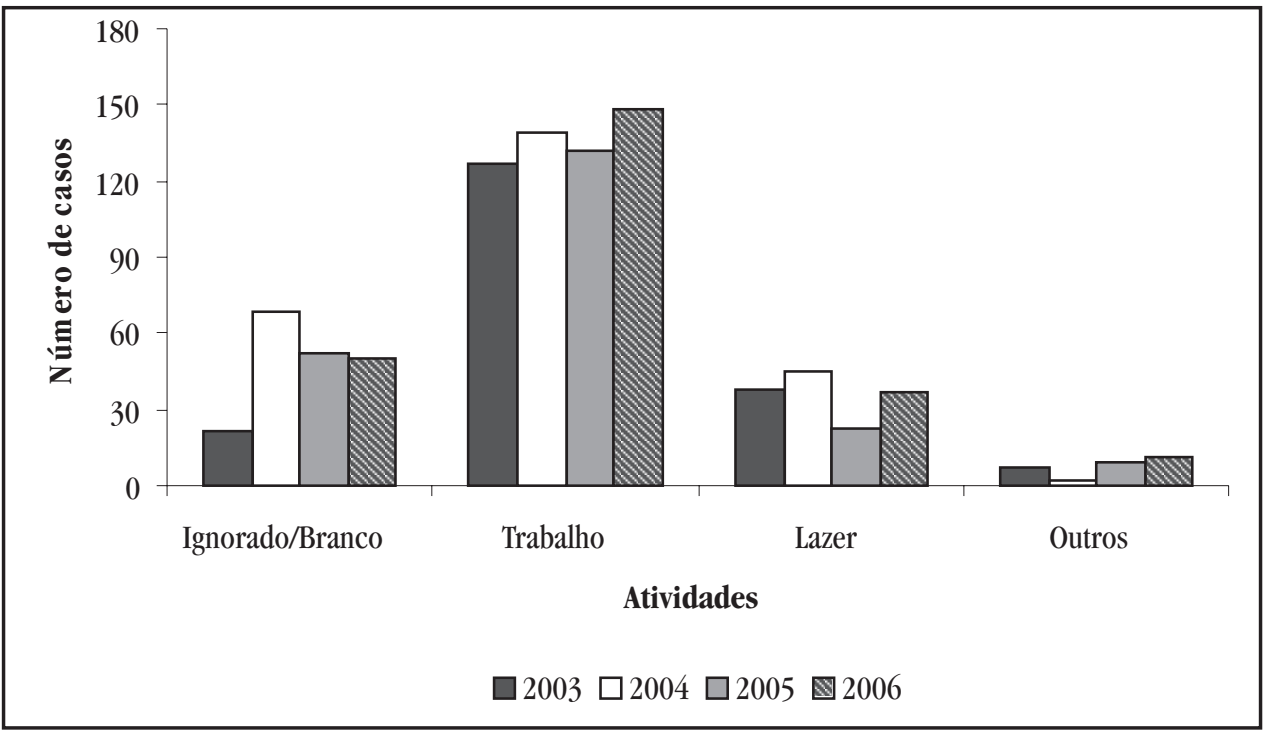

FIGURA 3

Frequência anual dos acidentes ofídicos no Estado do Amapá, segundo a circunstância do acidente, no período de 2003 a 2006.

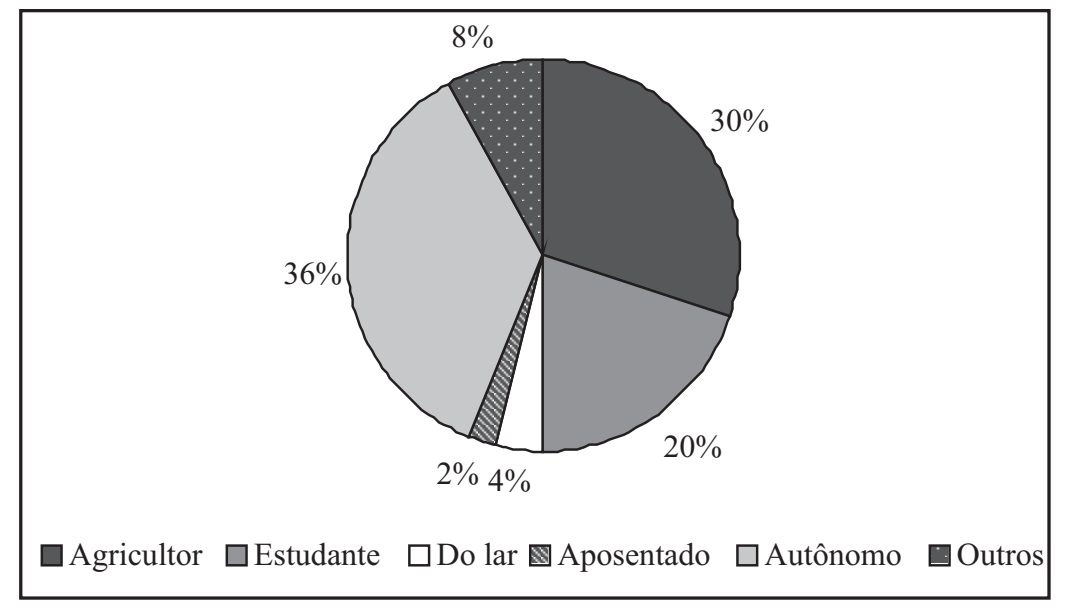

FIGURA 4

Distribuição dos acidentes ofídicos no Estado do Amapá, segundo o reflexo ocupacional, no período de 2003 a 2006. 
Em 625 casos que houve referência ao gênero da serpente que causou o acidente, 614 (67,5\%) foram por Bothrops, 6 (0,7\%) Crotalus e 3 (0,3\%) Lachesis. Nos registros, serpentes sem identificação de gênero ou sem preenchimento correto corresponderam a um total de 284 (31,2\%) dos acidentes (Figura 5).

As regiões anatômicas mais frequentemente atingidas foram os membros inferiores (68\%), seguidos por membros superiores (18\%), cabeça (3\%) e tronco (2\%). Em relação aos membros inferiores, as regiões das pernas (35\%) e pés (33\%) foram as mais atacadas. Quanto aos membros superiores, as mãos (15,9\%) foram as mais acometidas (Figura 6).
Os atendimentos aos acidentados ocorreram, em sua maioria em tempo superior a 12 horas após o acidente $(264$ casos) e, em 62 indivíduos o atendimento levou apenas 1 hora para acontecer (Figura 7).

Dos acidentes ofídicos, 263 (28,9\%) foram classificados como leves, seguidos por 193 (21,2\%) graves e 187 (20,6\%) moderados (Figura 8).

0 número de ampolas e soroterapia utilizadas foi de 5.244 ampolas, das quais 4.024 foram de soro botrópico, 1.178 de soro botrópico-laquético, 24 botrópico-crotálico, 14 crotálico e 4 elapídico (Figura 9).

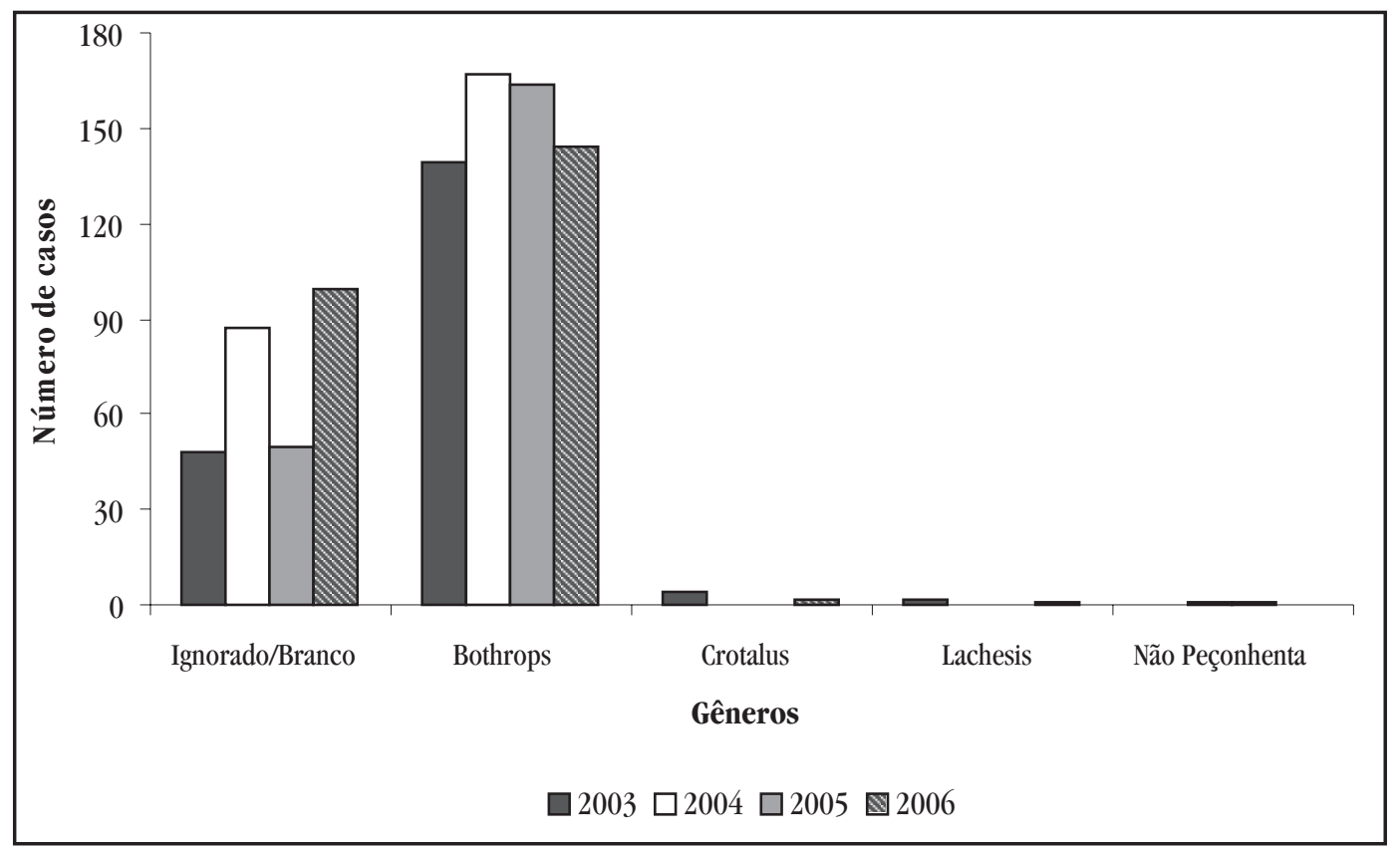

FIGURA 5

Frequência anual dos acidentes ofídicos no Estado do Amapá, segundo o gênero de serpente, no período de 2003 a 2006.

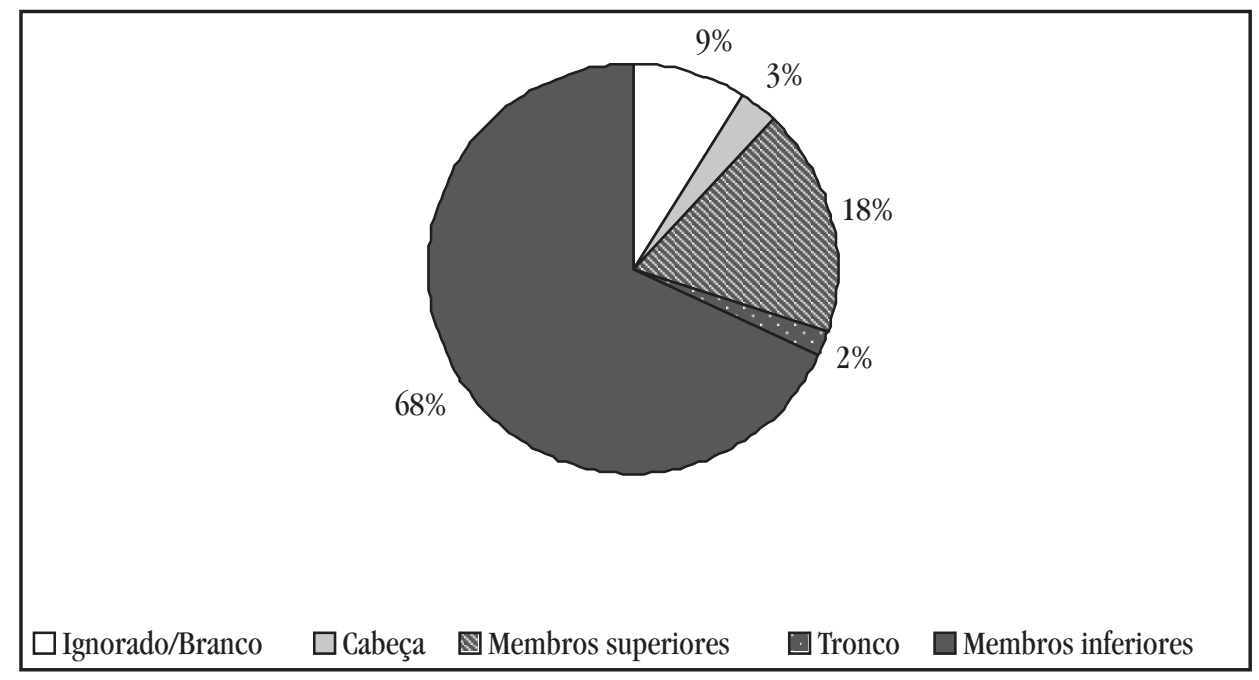

FIGURA 6

Frequência anual dos acidentes ofídicos no Estado do Amapá, segundo o local da picada, no período de 2003 a 2006. 


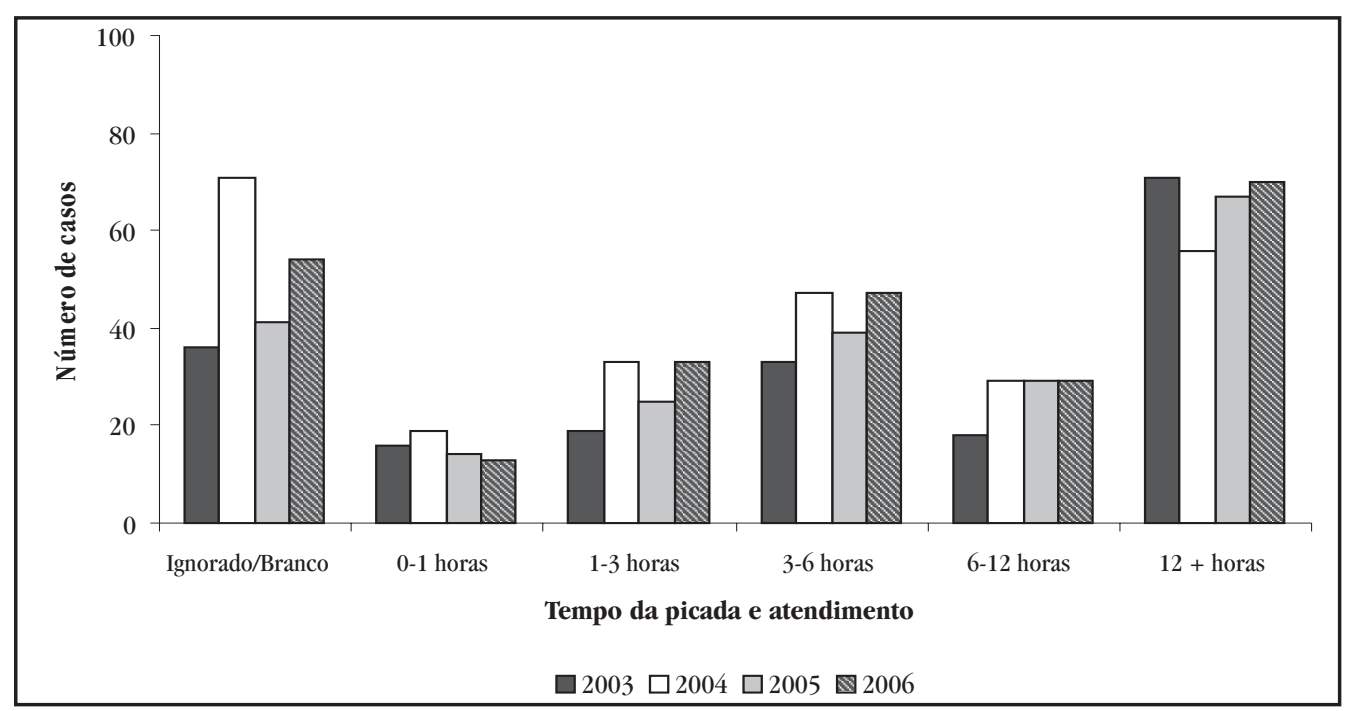

FIGURA 7

Frequência anual dos acidentes ofídicos no Estado do Amapá, segundo o tempo decorrido do momento do acidente até 0 atendimento médico, no período de 2003 a 2006.

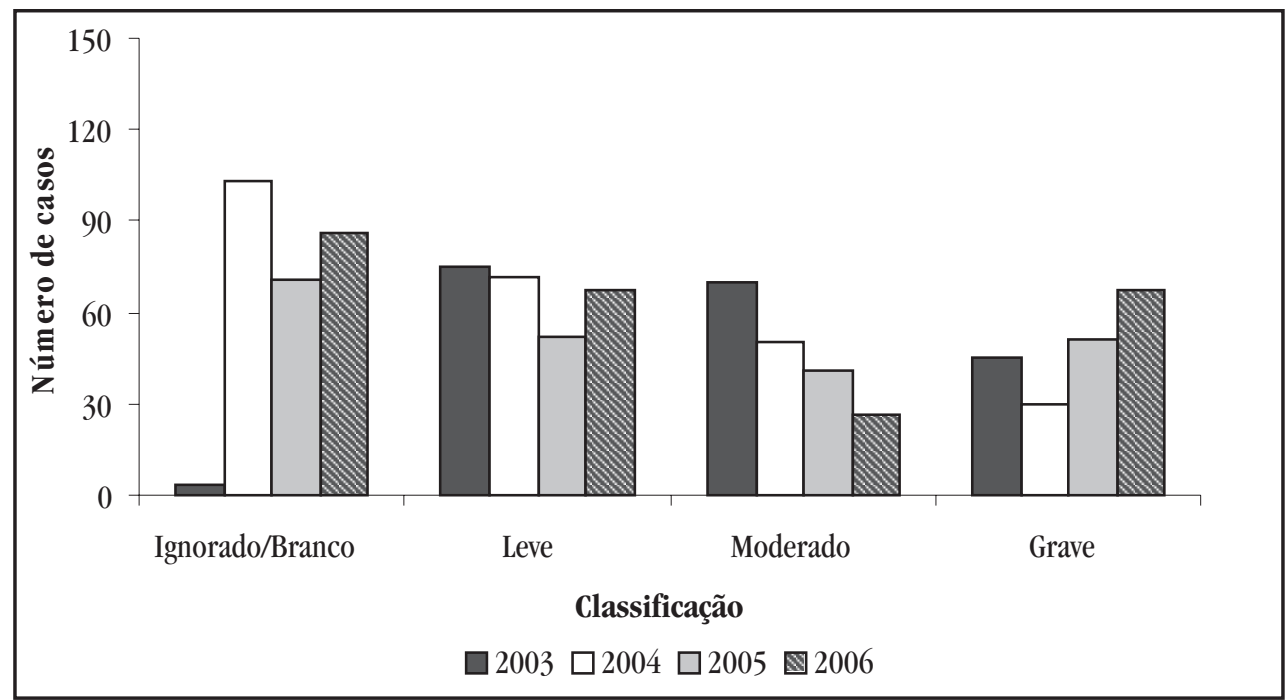

FIGURA 8

Frequência anual dos acidentes ofídicos no Estado do Amapá, segundo a classificação do acidente, no período de 2003 a 2006.

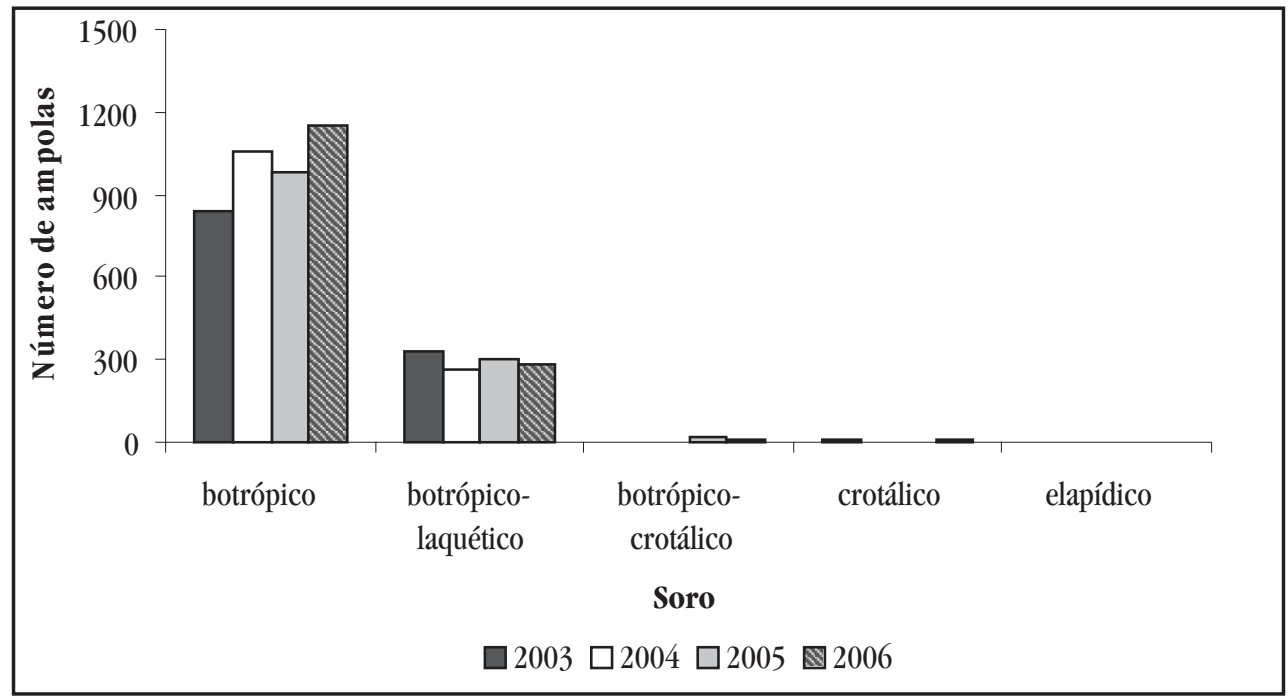

FIGURA 9

Frequência anual dos acidentes ofídicos no Estado do Amapá, segundo a soroterapia, no período de 2003 a 2006. 


\section{DISCUSSÃo}

O Estado do Amapá reproduz a expressiva ocorrência de número de acidentes ofídicos, semelhante ao do restante dos estados da região Norte ${ }^{17}$. Deste modo, a relevância do estudo dos acidentes ofídicos para o município de Macapá, é indicada pelo percentual de casos notificados para este município (909 casos).

A maioria dos acidentes envolveu indivíduos do sexo masculino, trabalhadores da zona rural, com idade entre 20 e 34 anos, seguindo o padrão apresentado em outros trabalhos realizados na região Norte, nos Estados do Amazonas ${ }^{6}$ e Roraima ${ }^{19}$. A provável causa de acidentes com este padrão é devido ao maior número de homens exercendo atividades que os expõem aos acidentes, como atividades extrativistas como caça, pesca e lavra da terra. Tais atividades rurais representam riscos para a ocorrência de acidentes ofídicos e que todos os outros dados relacionados ao sexo dos indivíduos e faixa etária parecem reforçar, ainda que de maneira sutil, a conotação deste tipo de acidente na maioria das vezes como um acidente de trabalho ${ }^{17102223}$.

A ocorrência de acidentes ofídicos nas zonas urbanas e rurais são notadamente distintos. Os casos registrados em áreas urbanas requerem especial atenção, demonstrando que as cidades necessitam de infra-estrutura necessária e adequada para que mantenham estes animais sem sinantropinização. Na zona urbana, 0 aumento na quantidade de resíduos domésticos produzidos e acondicionados de forma precária, principalmente nas áreas onde estão os bolsões de pobreza, atrai pequenos roedores cujo principal predador são as serpentes ${ }^{15}$.

Os membros inferiores e superiores foram as regiões anatômicas mais atingidas, concordando com os dados da literatura tanto para a região Norte ${ }^{61720}$ quanto para outras partes do país ${ }^{142123}$. A ausência de proteção adequada do indivíduo ao adentrar em matas ou ambientes comumente habitados por serpentes expõe partes do corpo a acidentes. Visto o maior número de acidentes atingirem as pernas, pés e mãos, é necessário o uso de botas, perneiras, luvas e demais vestimentas e equipamentos apropriados para prevenção de acidentes ${ }^{14}$.

0 tempo de atendimento após o acidente e a evolução dos casos ocorreu após 12 horas, devido às condições geográficas próprias da região amazônica (rios, estradas vicinais em péssimas condições, dependência de transporte fluvial ou terrestre). 0 tempo decorrido da picada e aplicação do soro é condicionante no sucesso de salvamento do paciente ${ }^{10}$. Na maioria $(68,5 \%)$ dos casos não houve a notificação da evolução do caso e 36,7\% evoluíram para a cura completa, havendo a notificação de óbito em apenas um $(0,7 \%)$, caso o que demonstra que os tratamentos adotados estão dentro dos padrões aceitáveis em si tratando de vidas humanas ${ }^{61720}$.

Os acidentes com o gênero Bothrops ocorreram durante todo período de estudo, sendo notificados 614 (67,5\%) acidentes. Apesar desse número ser inferior ao percentual notificado no país $(80 \%)^{13} 14$, está em conformidade com os dados obtidos em pesquisas regionalizadas ${ }^{3116}$, que podem indicar, porém, a existência de uma não notificação ou subnotificação dos acidentes uma vez que estes, em sua maioria, ocorrem em áreas rurais remotas cujas vitimas não tem facilidade no acesso aos postos de saúde. Os gêneros Lachesis e Crotalus apresentaram baixa incidência nos registros, fato observado para o Estado do Amazonas ${ }^{6}$. As serpentes do gênero Micrurus não figuram neste período com registro.

Inúmeros casos classificados como leve e moderados apontam para duas vertentes, a primeira, os leves denotam fragilidade na prescrição médica se realmente haveria necessidade de aplicação de soro espécie - específica (antiofídico) e a segunda, os moderados, requerem uma conduta médica específica em relação à quantidade de ampolas prescritas que podem estar aquém ou além da real necessidade que o caso requer. Estes números expressam a falta de parâmetros para o uso de soroterapia, caracterizado um desperdício geral e danos à saúde do acidentado ${ }^{129}$.

A maior incidência das picadas de serpentes do gênero Bothrops cuja média de ampolas por acidente é de 3,7 mostra uma classificação de acidente entre leve e moderado. Todavia, a carência de identificação exata da espécie de serpente conduz o médico a prescrever o imunobiológico bi-valente, ou seja, soro botrópico-laquético ou botrópico-crotálico ${ }^{825}$.

Vários fatores ambientais são apontados como variáveis que influenciam os acidentes ofídicos (e.g., clima, umidade, temperatura, pluviosidade), assim como 0 aumento de atividade humana no campo $0^{4}$. Entretanto, poucos trabalhos epidemiológicos relacionam os acidentes ofídicos com as atividades das serpentes. De um modo geral, essas atividades estão relacionadas à alimentação, termorregulação e, principalmente, reproduçã̃o $0^{1318}$.

\section{REFERÊNCIAS}

1. Albuquerque HN. Perfil clínico-epidemiológico dos acidentes ofídicos notificados no estado da Paraíba. Dissertação de Mestrado (Mestrado em Desenvolvimento e Meio Ambiente), Universidade Estadual da Paraíba, Universidade Federal da Paraíba, Campina Grande, 2002.

2. Amaral CFS, Dourado HV, Kouyoundjian JA, Cardoso JLC, Campos JA, AzevedoMarques MM, Lopes PFA. Manual de diagnóstico e tratamento de acidentes ofídicos, Ministério da Saúde, Brasília, 1991.

3. Aquino EK. Epidemiologia e clínica dos acidentes ofídicos no estado de Pernambuco. Dissertação de Mestrado (Mestrado em Biofísica), Departamento de Biofísica do Centro de Ciências Biológicas, Universidade Federal de Pernambuco, Recife, 1999.

4. Araujo FAA, SantaLucia M, Cabral RF. Epidemiologia dos acidentes por animais peçonhentos. In: Cardoso JLC, Siqueira França FO, Wen FH, Sant'ana Malaque CM, Haddad VJ (eds) Animais peçonhentos no Brasil: biologia, clínica e terapêutica dos acidentes. Editora Sarvier, São Paulo, p. 6-9, 2003.

5. BarravieraB, Ferreira Junior RS. Acidentes ofídicos. In: Focaccia R (ed) Veronesi Tratado de Infectologia. $3^{\text {a }}$ edição, Editora Atheneu, São Paulo, p. 1929-1947, 2005.

6. Borges CC, Sadahiro M, Santos MC. Aspectos epidemiológicos e clínicos dos acidentes ofídicos ocorridos nos municípios do Estado do Amazonas. Revista da Sociedade Brasileira de Medicina Tropical 32: 637-646, 1999.

7. Brazil VO. A defesa contra o ofidismo. Pocai-Weiss, São Paulo, 1911.

8. Cardoso JLC, Bucaretchi F, França FOS, Puorto G, Ribeiro LA, Azevedo-Marques MM, Jorge MT, Cupo P, Moraes RHP, Hering SE, Lucas SM, Gualtieri VBF. Acidentes por animais peçonhentos: identificação, diagnóstico e tratamento. Manual de Vigilância Epidemiológica. São Paulo, Secretaria de Estado da Saúde, 1993. 
9. Cardoso JLC, França FOS, Wen FH, Málaque CMSA, Haddad Júnior V. Animais peçonhentos no Brasil: Biologia, clínica e terapêutica dos acidentes. Editora Sarvier, São Paulo, 2003.

10. Carmo ICR. Contribuição para o estudo epidemiológico de acidentes ofídicos no Estado da Bahia no período de 1991-1992. Monografia (Bacharelado em Ciências Biológicas): Instituto de Biologia, Universidade Federal da Bahia, Salvador, 1994

11. Carvalho MA, Nogueira F. Serpentes da área urbana de Cuiabá, Mato Grosso: aspectos ecológicos e acidentes ofídicos associados. Cadernos de Saúde Pública 14: $753-763,1998$

12. Donato H. Achegas para a História de Botucatu. Botucatu, 1954.

13. Freitas MA, Silva TFS. Guia ilustrado: animais venenosos e peçonhentos no Brasil. Editora União Sul-Americana de Estudos da Biodiversidade, Porto Alegre, 2006

14. Fundação Nacional da Saúde. Manual de Diagnóstico e Tratamento de Acidentes por Animais Peçonhentos, Ministério da Saúde, Brasília, 2001.

15. Lima-Verde JS. Por que não matar as nossas cobras. In: Nascimento LB, Bernardes AT, Cotta GA (eds) Herpetologia no Brasil 1. Pontifica Universidade Católica/Biodiversitas, Belo Horizonte, p. 92-101, 1994.

16. Magalhães JS, Nascimento JFCG, Moura Júnior LA, Trajano SD, Trocolli RCP. Ofidismo: Experiência no Hospital Universitário Lauro Wanderley Durante 5 Anos (Setembro de 1986-Setembro de 1991). Revista Científica de Ciências da Saúde 8: 31-37, 1994.

17. Moreno E, Albuquerque MJ, Pires-de-Campos VAF, Katz G, Takaoka NY, Lebrão ML, Jorge MT. Características clínico epidemiológicas dos acidentes ofídicos em Rio Branco, Acre. Revista da Sociedade Brasileira de Medicina Tropical 38: 5-21, 2005.
18. Nascimento LB, Oliveira ME. Herpetologia no Brasil II. Sociedade Brasileira de Herpetologia, Belo Horizonte, 2007.

19. Nascimento SP. Aspectos epidemiológicos dos acidentes ofídicos ocorridos no Estado de Roraima, entre 1992-1998. Cadernos de Saúde Pública 16: 271-276, 2000

20. Pardal PPO, Monteiro MRC, Arnaund RN, Lopes FOB, Asano ME. Aspectos epidemiológicos de 465 acidentes ofídicos atendidos no HUJBB - Belém, Pará, no período de 1993 a 1994. Revista da Sociedade Brasileira de Medicina Tropical 28 (supl I): 170, 1995.

21. Pinho FMO, Pereira ID. Ofidismo. Revista da Associação Médica do Brasil 27 24-29, 2001

22. Resende CC, Araújo FA, Sallenave RNV. Análise Epidemiológica dos acidentes ofídicos do Brasil- Junho de 1986 a dezembro de 1987. Secretaria Nacional de Ações Básicas de Saúde, Ministério da Saúde, Brasília, 1989.

23. Ribeiro LA. Epidemiology of ophidic accidents. Memórias do Instituto Butantan 52 (supl): 15-16, 1991

24. Salomão MG, Albolea ABP, Almeida-Santos SM. Colubrid snakebite: a public health problem in Brazil. Herpetological Review 34: 307-312, 2003.

25. Souza IP. Alterações hematológicas e funcionais causadas por venenos de subespécies brasileiras de Crotalus durissus e suas frações isoladas. Tese de Doutorado (Doutorado em Farmacologia), Universidade Federal do Ceará, Fortaleza, 2006.

26. Warrel DA. Snakebites in Central and South America: epidemiology, clinical features, and clinical management. In: Campbell JA, Lamar WW (eds) The venomous reptiles of the Western Hemisphere. Cornell University Press, New York, p.709-715, 2004. 